\title{
Impact of contrast-enhanced CT and MRI on the management of patients with neurological diseases
}

\author{
Karl-Olof Lovblad
}

Even though a thorough clinical examination by a welltrained neurologist can very often determine precisely the extent of neurological deficits in a given patient, additional information is often needed to optimize treatment. Indeed, as many disease entities are becoming treatable due to improvements in pharmacology, surgical techniques and endovascular methods, we as neuroradiologists must position ourselves more clearly and be able to provide acutely precise information regarding not just the nature of the lesion but also the potential outcome of any given treatment. The main areas that come to mind are cerebrovascular diseases, brain tumors [1] and inflammatory diseases where contrast agents have greatly improved our insight into the acute pathological processes that occur in the diseased brain. While we as radiologists are aware of the importance of these techniques, we have to further explore their implications on patient management and outcome in order to position ourselves in relation to other physicians as indispensable partners in the ongoing clinical decision-making process.

Brain imaging was initially revolutionized by the development of CT. This was succeeded by MRI. These two techniques have over the last two decades continued to evolve and have become invaluable tools for the management of patients with neurological diseases. As regards cerebrovascular diseases, both MRI and CT have undergone important evolutionary steps over the last few years due to the widespread availability of perfusion techniques. Simi-

K. O. Lovblad ( $\square)$

Neuroradiology Unit, Radiology Department,

HUG, Geneva University Hospital,

24 Micheli-du-Crest,

1211 Geneva, Switzerland

Tel.: +41-22-3727033

Fax: +41-22-3727072

e-mail: karl-olof.lovblad@hcuge.ch larly, with the emergence of high-powered MR gradients and MR angiography, it became possible to perform neurovascular imaging from the aortic arch to the circle of Willis in one session [2]. The advent of diffusion techniques, perfusion imaging and MR spectroscopy also led to improvements in MRI; these improvements have themselves been improved by the continual increase in magnetic field strength over the years. More recently, with the advent of faster spiral scanners and with the development of multirow detectors, CT technology has caught up and now also allows neuroangiographic examinations to be performed that rival conventional digital subtraction angiographic techniques.

Once these technological hardware considerations had been addressed, it was obvious that contrast media would also be able to contribute to the improvement in image quality and thus finally to improved patient management. This we were able to see at a satellite symposium organized by Bracco during the 2006 Meeting of the ESNR (13-16 September 2006, Geneva, Switzerland). Dr. T. Krings (Aachen, Germany) demonstrated that the use of improval MR contrast agents can provide images of the intra- and extracranial carotids of very high quality, and this obviously has an important impact on patient management. Indeed, in less than a decade, we have seen how MRA has established itself as a valid alternative to conventional digital subtraction techniques for the work-up of patients with cerebrovascular diseases. CT techniques can also be improved by the use of high-concentration iodinated contrast media in the carotid system with an important impact on decisions regarding therapy, as was explained by Dr. B. Schuknecht (Zurich, Switzerland). Dr. P. Picozzi (Milan, Italy) demonstrated how MRI with gadobenate dimeglumine (MultiHance) can help detect lesions in a way that has major implications for both neurosurgical planning and follow-up. Parallel to this, 
Dr. P. Schramm (Heidelberg, Germany) demonstrated that the care of neurosurgical patients can also be improved with the use of high-concentration iodinated contrast media.

As we can see from the papers in this supplement to Neuroradiology, the advent of new contrast media allows us as neuroradiologists to affect patient management and outcome safely and reliably. This is an area in which we can contribute together with our partners in the equipment and contrast agent industries. Indeed, the development of newer more powerful contrast agents such as gadobenate dimeglumine for MRI has allowed us to investigate with increased detail the diseased nervous system and thus to gain further knowledge leading to improved patient management.

\section{References}

1. Cotton F (2006) Diffusion-perfusion in intra-axial brain tumors with high relaxivity contrast agents. Neuroradiology 48 [Suppl 1]: 34-40

2. Remonda L, Senn P, Barth A et al (2002) Contrast-enhanced 3D MR angiography of the carotid artery: comparison with conventional digital subtraction angiography. AJNR Am J Neuroradiol 23(2):213-219 